\title{
The effect of lactose-free formula feeds on growth responses among severely malnourished HIV- infected children in Durban, South Africa
}

\author{
E Binka, ${ }^{1} \mathrm{MD}$; D Montoya-Fontalvo, ${ }^{1} \mathrm{MD} ; \mathrm{M}$ Healy, ${ }^{1} \mathrm{MD} ;$ M Sobieszczyk, ${ }^{1} \mathrm{MD}, \mathrm{MPH}$; P LaRussa, ${ }^{1} \mathrm{MD}$; \\ R Bobat, ${ }^{2} \mathrm{MB}$ ChB, FCPaed, MD; $\mathbf{M}$ Archary, ${ }^{2} \mathrm{MB}$ ChB, DCH (SA), FCPaeds, Paeds ID (SA) \\ Department of Medicine, Columbia University College of Physicians and Surgeons, Columbia University, New York, USA \\ ${ }^{2}$ Paediatric Infectious Diseases Unit, Department of Paediatrics and Child Health, Nelson R Mandela School of Medicine, University of \\ KwaZulu-Natal, Durban, South Africa
}

Corresponding author: M Archary (archary@ukzn.ac.za)

\begin{abstract}
Background. The co-occurrence of HIV infection and severe malnutrition contributes to high rates of morbidity and mortality among children in resource-limited settings. Lactose-free, ready-to-use therapeutic feeds (RUTFs) may be most appropriate in this population because of underlying mucosal damage secondary to inflammation and infection.

Objectives. To describe the effect of lactose-free RUTFs on the growth parameters of severely malnourished HIV-infected children in Durban, South Africa (SA).

Methods. This was a prospective, observational study of nutritional recovery in HIV-infected, severely malnourished children, aged 6 months to 5 years, who received lactose-free RUTFs following admission to King Edward VIII Hospital in Durban, SA. The primary outcome was nutritional recovery, defined as $15 \%$ weight gain from enrolment to end of study. Secondary outcomes included $z$-scores for weight-for-height, weight-for-age, height-for-age, triceps skinfold thickness (SFT) and subscapular SFT calculated at baseline and 7, 14, 30 and 45 days after admission. Univariate analysis was done to compare outcomes among antiretroviral therapy (ART)-naive and ARTexperienced children; the effect of ART on nutritional recovery was evaluated in a logistic regression model.

Results. A significant improvement in most nutritional parameters was found at 45 days; $59 \%$ of children attained nutritional recovery. There was no significant difference in the proportion of children reaching recovery based on ART status at admission $(p=0.08)$.

Conclusion. Lactose-free formula feeds may be an effective strategy for nutritional rehabilitation of severely malnourished and HIVinfected children in resource-limited settings. It remains to be determined how ART initiation affects nutritional recovery in these children.
\end{abstract}

S Afr J Child Health 2015;9(4):130-132. DOI:10.7196/SAJCH.2015.v9i4.814

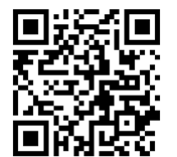

Severe acute malnutrition (SAM) affects 13 million children under the age of 5 years and leads to 1 - 2 million preventable paediatric deaths annually. ${ }^{[1]}$ The World Health Organization (WHO) defines SAM as the presence of at least one of: $(i)$ weight-for-height more than $70 \%$ below the age-specific median or three or more standard deviations (SDs) below the mean in a normally distributed population; (ii) mid-upper arm circumference $<115 \mathrm{~mm}$; or (iii) the presence of bilateral lower extremity pitting oedema of nutritional origin. ${ }^{[2,3]}$

In developing countries, there is often an overlap between HIV infection and malnutrition. The prevalence of SAM is approximately three-fold higher in HIV-infected than HIV-uninfected children, ${ }^{[4]}$ and SAM is often the precipitating event that prompts healthcareseeking behaviour among HIV-infected children. Co-occurrence of SAM and HIV increases morbidity, including stunted physical and cognitive development, and mortality. ${ }^{[5,6]}$

The WHO recommends the use of two specific formula diets as treatment for SAM: the F-75 (75 kcal-th or $315 \mathrm{~kJ} / 100 \mathrm{~mL}$ ) during the initial phase of treatment and the F-100 (100 kcal-th or $420 \mathrm{~kJ} / 100 \mathrm{~mL}$ ) during the rehabilitation phase after the child's appetite has returned. ${ }^{[2]}$ Countries such as South Africa (SA) have developed local, ready-to-use therapeutic feeds (RUTFs) similar in nutritional composition to the WHO-recommended F-100 formula. Unlike the F-100, RUTFs are not water based, thus minimising potential contamination or barriers associated with water acquisition. ${ }^{[6,7]}$ Furthermore, the WHO-recommended feeds used to treat SAM are high in lactose and may not be optimal for HIV-infected children who tend to present with diarrhoea and malabsorption. Few studies have investigated the effect of the local and lactose-free RUTFs on the growth responses and nutritional recovery of severely malnourished HIV-infected children. ${ }^{[8]}$ Our study describes the effect of a lactose-free RUTF on growth responses of severely malnourished HIV-infected children in Durban, SA.

\section{Methods \\ Patients}

Children admitted to the King Edward VIII Hospital paediatric wards were screened during two time periods: between February and March 2011 and between December 2011 and April 2012. Those who were 6 months - 5 years of age, confirmed by HIV DNA polymerase chain reaction or HIV ELISA to be HIV-infected, and met the WHO definition of SAM, were eligible for enrolment in this prospective, observational study. Antiretroviral (ARV) therapy (ART)-experienced children were defined as children who reported taking ARVs at the time of admission; ART-naive children were defined as children who were not taking ARVs at admission. All patients were fed with an age-appropriate, lactose-free RUTF based on the postadmission day (Table 1).

\section{Study procedures}

Parents and guardians were approached to obtain written informed consent. The consent form was read to them in English or IsiZulu with the use of a translator. Measurements of weight, height, and biceps, triceps, subscapular and mid-thigh skinfold thickness (SFT), and demographic and pertinent clinical information were obtained at baseline and at days 7, 14, 30 and 45 post enrolment. SFT measurements were done with a Harpenden Skinfold Caliper (Baty International, UK). The study was approved by the Institutional Review Board of Columbia University and the Biomedical Research 
Ethics Committee at the University of KwaZulu-Natal, as well as by the hospital management.

\section{Statistical analysis}

Indices of growth response and recovery were calculated using the WHO Anthro software (version 3.2.2). Z-scores for weight-for-height (WHZ), weight-for-age (WAZ), height-for-age (HAZ), triceps SFT (TSZ) and subscapular SFT (SSZ) were calculated at baseline and at follow-up days 7, 14, 30 and 45 . Nutritional recovery was defined as $\geq 15 \%$ weight gain from enrolment through to end of study period or by day 45. Data were captured in Microsoft Access (USA). A binary logistic regression model was applied to data, including age, gender, CD4 cell count and percentage, baseline weight, ART status at baseline, presence of pedal oedema and WHZ $>70 \%$ as independent variables. Analysis was performed using SPSS version 19 (IBM, USA).

\section{Results}

Baseline demographics of participants are shown in Table 2. Sixteen of 30 children had CD4\% available at baseline. Of these, $12(75 \%)$ were severely immunosuppressed (CD $4 \%<15 \%)$. Of 30 children, eight had HIV-1 viral loads available at baseline; median $\log _{10} \mathrm{HIV}-1$ RNA was 6.3 (interquartile range (IQR) 5.7 - 6.9). Fifteen out of 30 children (50.0\%) were on ARVs at enrolment.

Table 1. SAM refeeding protocol

\begin{tabular}{lll}
\hline Days post admission & Energy $(\mathrm{kcal} / \mathrm{kg})$ & Protein $(\mathrm{g} / \mathrm{kg})$ \\
\hline $1-2$ & $33-37$ & 1.0 \\
$3-4$ & $50-56$ & 1.5 \\
$5-6$ & $67-74$ & 2.0 \\
7 & $83-93$ & 2.5 \\
$>7$ & $100-112$ & 3.0
\end{tabular}

Table 2. Baseline demographics and growth parameters in 30 HIV-positive children before starting treatment with a ready-to-use lactose formula feed

\begin{tabular}{ll}
\hline Variable & HIV-positive $(n=30)$ \\
\hline Age (months), mean (SD, IQR) & $16.1(12.0,7.6-18.9)$ \\
Gender (male), \% $(n)$ & $46.7(14)$ \\
CD4\%, mean (SD) $(n=16)$ & $14.1(11.2)$ \\
CD4 $<15 \%, \%(n)$ & $75(12)$ \\
HIV-1 RNA, log 10 median (IQR) $(n=8)$ & $6.3(5.7-6.9)$ \\
Weight (kg), mean (SD) & $6.2(1.5)$ \\
Height (cm), mean (SD) & $69.2(7.8)$ \\
$\begin{array}{l}\text { Duration of follow-up (days), } \\
\text { median (IQR) }(n=28)\end{array}$ & $44.0(27-45)$ \\
On ART ${ }^{*}$ at study start, \% $(n)$ & $50(15)$ \\
Time on ART at study start (months), & $2.0(1.5-3)$ \\
median (IQR) $(n=9)$ & \\
WAZ, mean (SD) & $-3.97(1.0)$ \\
WHZ, mean (SD) & $-3.27(1.1)$ \\
Mid-upper arm circumference & $28.0(93.3)$ \\
$(\leq 115$ mm), $n(\%)$ & \\
TSZ, mean (SD) & $-3.08(1.2)$ \\
SSZ, mean (SD) & $-3.95(1.7)$ \\
${ }^{*}$ Children $<3$ years were on abacavir, lamivudine and lopinavir/ritonavir. Children \\
$>3$ years were on abacavir, lamivudine and efavirenz.
\end{tabular}

Baseline growth parameters for participants are shown in Table 2. Mean baseline growth parameters indicated that the children were severely malnourished: mean (SD) WAZ -3.97 (1.0), mean WHZ -3.27 (1.1), mean TSZ -3.08 (1.2) and mean SSZ -3.95 (1.7). Twentyfour of 30 had a WHZ $<70 \%$ of the median, suggesting that this was more of an acute presentation of severe malnutrition rather than a chronic state where stunting would be more prevalent. Baseline comorbidities are shown in Table 3.

Twenty-two of the 30 children had evaluations through to study end at day 45 . Three died of opportunistic infections and five were lost to follow-up. Of the study subjects, $59.0 \%(n=13)$ attained nutritional recovery while $41.0 \%(n=9)$ remained malnourished. There was a significant improvement from baseline to day 45 ( $n=22$ patients) in WAZ (1.34 (1.90), $p=0.003)$, TSZ (1.43 (1.60), $p=0.0005)$ and SSZ (2.02 (2.50), $p=0.001)$, a trend to significance for WHZ (0.95 (2.20), $p=0.060)$ and a statistically insignificant improvement for HAZ $(0.76$ (0.50), $p=0.100)$. The mean rate of weight gain was 3.5 (6.9) g/kg/day but increased to $4.7(5.0) \mathrm{g} / \mathrm{kg} /$ day after excluding the three deceased.

A comparison of baseline characteristics and clinical outcomes between ART-experienced and ART-naive children at the start of the study showed no significant differences (Table 4). However, in univariate analysis, ART-naive children had a slightly higher likelihood of reaching nutritional recovery at day 45 (60\% v. $40 \%$; $p=0.08)$ than children who were ART-experienced. In logistic regression analysis, being ART-naive at presentation was associated with nutritional recovery at day 45 (OR 2.25, $p=0.465$ ), although this was not statistically significant (Table 5). Demographic factors, baseline weight, and CD4 cell percentage were not associated with nutritional recovery at day 45 .

\section{Discussion}

Current WHO guidelines for the treatment of SAM do not make specific formula recommendations for refeeding children who are both severely malnourished and HIV-infected. With HIV infection, there is mucosal damage secondary to infection or inflammation leading to chronic diarrhoea, protein-losing enteropathy, carbohydrate deficiencies and fat malabsorption. ${ }^{[7]}$ Therefore, lactose-free formulas may be appropriate alternatives for refeeding regimens in this population. Our results showed that HIV-infected children on lactosefree RUTFs gained an average of $3.5 \mathrm{~g} / \mathrm{kg} /$ day. This figure is similar to the average weight gain of severely malnourished children known to be HIV-infected in previous studies of lactose-containing formulas recommended by the WHO. Ndekha et al. ${ }^{[9]}$ and Sandige et al. ${ }^{[10]}$ studied children in Malawi who were severely malnourished and HIVinfected; both studies reported an average weight gain of $3.1(2.7) \mathrm{g} / \mathrm{kg} /$ day and $3.6(4.7) \mathrm{g} / \mathrm{kg} /$ day, respectively. Our study showed that children refed with lactose-free RUTF gained weight in a similar fashion. However, our study was limited owing to policies of the institution that did not make it possible to directly compare lactose-free with lactose-

Table 3. Baseline comorbidities in $30 \mathrm{HIV}$-positive children

\begin{tabular}{ll}
\hline Comorbidity & HIV-positive, \% $(n)(N=30)$ \\
\hline Diarrhoea & $23(7)$ \\
Pulmonary tuberculosis & $17(5)$ \\
Abdominal tuberculosis & $3(1)$ \\
Urinary tract infection & $3(1)$ \\
Pneumonia & $13(4)$ \\
Dermatitis & $13(4)$ \\
Oral thrush & $13(4)$ \\
Cytomegalovirus & $3(1)$ \\
Toxoplasmosis & $3(1)$
\end{tabular}


Table 4. A comparison of baseline characteristics and clinical outcomes in ARTexperienced and ART-naive children receiving ready-to-use lactose-free formula feeds

\begin{tabular}{llll}
\hline & ART-experienced $(N=15)$ & ART-naive $(N=15)$ & $p$-value \\
\hline Baseline characteristics & & & \\
Age (months), mean (SD, IQR) & $17.6(11.9,9.4-21.9)$ & $14.7(12.3,7-17)$ & 0.56 \\
CD4 (\%), mean (SD) & $20.66(13.64)(n=9)$ & $9.03(5.24)(n=7)$ & 0.34 \\
HIV-1 viral load, mean (SD) & $5.7(2.1)(n=5)$ & $6.1(0.6)(n=3)$ & 0.73 \\
$\begin{array}{l}\text { Weight (kg), mean (SD) } \\
\text { End of study outcomes (day 45) }\end{array}$ & $6.6(1.7)$ & $5.7(1.3)$ & 0.15 \\
$\begin{array}{l}\text { Proportion reaching } \\
\text { nutritional recovery (\%) }\end{array}$ & 40 & 60 & 0.08 \\
$\begin{array}{l}\text { Rate of weight gain } \\
\text { (g/kg/day), mean (SD) }\end{array}$ & $2.63(2.7)$ & $4.39(9.4)$ & 0.49 \\
$\begin{array}{l}\text { Change in WHZ, mean (SD) } \\
\text { Change in WAZ, mean (SD) }\end{array}$ & $-0.54(4.1)$ & $1.45(0.22)$ & 0.12 \\
Change in HAZ, mean(SD) & $0.64(1.2)$ & $1.29(1.7)$ & 0.19 \\
& & $0.93(0.9)$ & 0.53
\end{tabular}

Table 5. Logistic regression model ${ }^{\star}$ evaluating effect of concurrent ART on nutritional recovery at 45 days

\begin{tabular}{lllll}
\hline ART at baseline & $\begin{array}{l}\text { Univariate OR } \\
(\mathbf{9 5 \%} \text { CI })\end{array}$ & $p$-value & $\begin{array}{l}\text { Multivariate OR } \\
(\mathbf{9 5 \% ~ C I ) ~}\end{array}$ & $p$-value \\
\hline Yes & $0.44(0.10-1.920$ & 0.465 & $0.54(0.08-3.89)$ & 0.54 \\
No & $2.25(0.52-9.70)$ & Ref & $1.86(0.26-13.40)$ & Ref
\end{tabular}

${ }^{\star}$ Logistic regression model included age, gender, CD4 cell count, CD4\%, baseline weight, baseline ART status, presence of pedal oedema and $\mathrm{WHZ}>70 \%$ (independent variables), and nutritional recovery (dependent variable).

containing formulas. We were also limited by our inability to confirm the presence of lactose maldigestion in our patients at presentation.

The WHO recommends that the minimum rate of weight gain at the end of a nutritional rehabilitation period should be $5.0 \mathrm{~g} / \mathrm{kg} /$ day. After excluding values for the deceased children, our mean weight gain increased to $4.7 \mathrm{~g} / \mathrm{kg} /$ day, approaching WHO-expected rates. This occurred despite the expectation that severely malnourished children who are HIV-infected should gain less weight or gain less rapidly than a normally distributed population, given the malabsorptive state and mucosal damage created by HIV infection.

Even when using high-energy RUTFs to manage SAM in HIV-infected children, mortality rates of $\sim 38 \%$ at $4-6$ weeks are too high. ${ }^{[11]}$ Our mortality rates were slightly lower. At the end of our 45-day study period, three patients had died (10\% of our study population). Although the use of RUTF diets has been shown to lead to significant and sustained weight gain in HIV-infected children, this improvement has not always been associated with recovery in CD4 cell count or improvement in survival. ${ }^{[11,12]}$ One major limitation of our study was that we were not able to track the changes in CD4 cell count and percentage in our patients to determine if there was any association between nutritional recovery and improvement in CD4 cell count or percentage. types of formulas in promoting nutritional recovery and explore how the timing of ART initiation with respect to acute refeeding affects nutritional recovery in these children.

\section{References}

1. Collins S, Dent N, Binns P, Bahwere P, Sadler K, Hallam A. Management of severe acute malnutrition in children. Lancet 2006;368(9551):1992-2000. [http://dx.doi.org/10.1016/S0140-6736(06)694439]

2. World Health Organization. Management of severe malnutrition: A manual for physicians and other senior health workers. Geneva: World Health Organization, 1999.

3. World Health Organization/World Food Programme/United Nations System Standing Committee on Nutrition/United Nations Children's Fund Community-based management of severe acute malnutrition. Geneva/Rome/Geneva/New York World Health Organization/World Food Programme/ United Nations System Standing Committee on Nutrition/United Nations Childrenss Fund, 2007.

4. Prendergast A, Bwakura-Dangarembizi MF, Cook $\mathrm{AD}$, et al. Hospitalization for severe malnutrition among HIV-infected children starting antiretroviral therapy. AIDS 2011;25(7):951-956. [http://dx.doi. org/10.1097/QAD.0b013e328345e56b]

5. Chinkhumba J, Tomkins A, Banda T, Mkangama C, Fergusson P. The impact of HIV on mortality during in-patient rehabilitation of severely malnourished children in Malawi. Trans R Soc Trop Med Hyg 2008;102(7):639-644. [http:// dx.doi.org/10.1016/j.trstmh.2009.04.02]

6. Miller T, Orav EK, Martin SR, Cooper ER, McIntosh K, Winter HS. Malnutrition and carbohydrate malabsorption in children with vertically transmitted human immunodeficiency virus 1 infection. Gastroenterol 1991;100(5):1296-1302.

7. Hendricks KM. Ready-to-use therapeutic food for prevention of childhood undernutrition. Nutr Rev 2010;68(7):429-435. [http://dx.doi.org/10.1111/ j.1753-4887.2010.00302.x]

8. Biggs C. Clinical dietetic practice in the treatment of severe acute malnutrition in a high HIV setting. J Hum Nutr Diet 2013;26(2):175-181. [http:// dx.doi.org/10.1111/jhn.12003]

9. Ndekha MJ, Manary MJ, Ashorn P, Briend A. Home-based therapy with ready-to-use therapeutic food is of benefit to malnourished, HIV-infected Malawian children. Acta Paediatr 2005;94(2):222-225.

10. Sandige H, Ndekha MJ, Briend A, Ashorn $P$ Manaray MJ. Home-based treatment of malnourished Malawian children with locally prod uced or imported ready-to-use food. J Pediatr Gastroenterol Nutr 2004;39(2):141-146.

11. Heikens GT, Bunn J, Amadi B, et al. Case management of HIV-infected severely malnourished children: Challenges in the area of highest prevalence. Lancet 2008;371(9620):1305-1307. [http://dx.doi org/10.1016/S0140-6736(08)60565-6]

12. Rollins NC, van den Broeck J, Kindra G, Pent M, Kasambira T, Bennish ML. The effect of nutritional support on weight gain of HIVinfected children with prolonged diarrhoea. Acta Paediatr 2007;96(1):62-68.

13. Kim MH, Cox C, Dave A, et al. Prompt initiation of ART with therapeutic food is associated with improved outcomes in HIV-infected Malawian children with malnutrition. Acquir Immune Defic Syndr 2012;59(2):173-176. [http://dx.doi. org/10.1097/QAI.0b013e3182405f8f

14. Sutcliffe CG, van Dijk JH, Munsanje B, et al. Weight and height $z$-scores improve after initiating ART among HIV-infected children in rural Zambia: A cohort study. BMC Infect Dis 2011;11(1):54. [http:// dx.doi.org/10.1186/1471-2334-11-54]

15. Weigel R, Phiri S, Chiputula F, et al. Growth response to antiretroviral treatment in HIV-infected children A cohort study from Lilongwe, Malawi. Trop Med Int Health 2010;15(8):934-944. [http://dx.doi org/10.1111/j.1365-3156.2010.02561.x ]
Lactose-free formula feeds may be an effective strategy for nutritional rehabilitation of severely malnourished and HIV-infected children in resource-limited settings. Future studies should directly compare the effectiveness of both 